\title{
Detonation Characteristics of
}

\section{Hydrogen-Oxygen Mixtures at}

\section{High Initial Pressures}

\author{
ROY L. GEALER and STUART W. CHURCHILL \\ The University of Michigan, Ann Arbor, Michigan
}

\begin{abstract}
Detonation velocities of hydrogen-oxygen mixtures were measured for initial pressures from 14.4 to $1,000 \mathrm{lb} . / \mathrm{sq}$. in. abs, and compositions from 40 to 80 mole \% molecular hydrogen. Detonation and impact characteristics were computed for essentially the same range of conditions by the use of the elementary theory of detonation. Equilibrium compositions of hydrogen-oxygen mixtures at pressures from 10 to $2,000 \mathrm{~atm}$. and temperatures from 3,000 to $5,000^{\circ} \mathrm{K}$. were included in the computations and have been filed with the American Documentation Institute. The effect of nonideal thermodynamic properties on the computed detonation velocities was considered.
\end{abstract}

Since the velocity of propagation of a detonation wave depends upon the energy released in the combustion process, and since the net energy released is diminished by the energy consumed in dissociating the products of combustion, a decrease in the degree of dissociation of the products should result in an increase in detonation velocity. In the hydrogen-oxygen system an increase in the pressure of the system decreases the degree of dissociation of the products of combustion and thus can be expected to increase the velocity of the detonation wave.

Detonation velocities have been measured at nonambient conditions by only a few investigators. Dixon (3) determined detonation velocities in stoichiometric mixtures of hydrogen and oxygen at $10^{\circ}$ and $100^{\circ} \mathrm{C}$. over a pressure range from 200 to $1,500 \mathrm{~mm}$. Hg. Moyle, Morrison, and Churchill (18) measured velocities and computed theoretical detonation characteristics over a temperature range from $160^{\circ}$ to $480^{\circ} \mathrm{K}$. and a pressure range from 0.5 to $2 \mathrm{~atm}$. in various hydrogenoxygen mixtures. Hoelzer and Stobaugh (10) measured velocities in hydrogenoxygen and ethane-oxygen mixtures over a pressure range from 1 to $10 \mathrm{~atm}$.

Roy L. Gealer is with the Ethyl Corporation, Detroit, Michigan. for several compositions. The purpose of this investigation was to measure detonation velocities in hydrogen-oxygen mixtures over a considerably extended range of pressure.

Theoretical detonation characteristics were also computed for the experimental conditions by the use of the elementary theory of detonation. Since this work was completed, Luker, McGill, and Adler (I7) have reported theoretical characteristics, for stoichiometric mixtures only, over the same range of pressures.

\section{EXPERIMENTAL EQUIPMENT AND PROCEDURE}

Figure 1 shows a sketch of the equipment. A 2-1/2-in., double-extra-heavy steel pipe was used as a mixing and pressurizing chamber. After evacuation a mixture of approximately the desired composition was proportioned into the vertical mixing pipe from the gas bottles by partial pressure. Fire resistant hydraulic fluid was pumped up through the bottom of the pipe to compress the mixture to the desired pressure. The pressurized gas was then forced out the tip of the mixing tube and into the bottom of the vertical detonation tube. Samples of each mixture were analyzed by absorbing the oxygen in a solution of alkaline pyrogallol and measuring the volumetric loss at constant pressure. Three analyses were made for each sample, and the arithmetic average was used. Samples were taken at several different times to establish the minimum time required for mixing.

The stainless steel detonation tube had a 4-in. O. D. and a 1/2-in. I. D. It was mounted vertically with a hot-wire ignitor near the bottom and ionization probes for velocity measurement spaced 18 in. apart, near the top. The first probe was located 53 in. from the ignitor, since the data of Dumanois and Lafitte (4) indicated that a fully developed wave velocity would be obtained in this distance. Velocity measurements at a series of probe locations confirmed that this distance was sufficient except in rich mixtures at low pressures as discussed later.

After the high pressure mixture of hydrogen and oxygen was charged into the detonation tube, which was closed at the top by a brass rupture diaphragm, the tube was closed off from the rest of the system and the mixture ignited. As a detonation wave passed through the tube; one ionization probe and thyratron circuit started a timer, and a second stopped it. The timer registers time intervals down to $0.1 \mu \mathrm{sec}$. with an accuracy of $\pm 0.1 \mu \mathrm{sec}$. The $100 \mathrm{kc}$. oscillator on the timer was standardized by beating the output against National Bureau Standards radio station WWV, the crystal being adjusted until zero beat was obtained.

The detonation tube and auxiliary equipment were located in a deep concrete-lined pit in a special test cell isolated from the laboratory area by a $1 \mathrm{ft}$.- 
thick, poured-concrete wall containing a bullet-proof glass window. All valves were operated remotely by means of flexible push-pull cable controls attached to levers on the valves, and the pressure gauges were viewed through the bullet-proof window by means of a mirror and telescope arrangement. Destruction of the charging system by an accidental explosion substantiated these precautions. Additional details concerning the equipment and work are given in reference 6.

\section{PREDICTION OF DETONATION CHARACTERISTICS}

The detonation characteristics of hydrogen-oxygen mixtures were computed for the experimental range of conditions by the use of the elementary theory of detonation. This theory postulates a planar discontinuity in pressure, temperature, and composition moving at sonic velocity relative to the burned gases (the Chapman-Jouguet condition); chemical and thermal equilibrium in the burned gases; no longitudinal energy, component, or momentum transfer except by flow; no transfer to the surroundings; and ideal gas behavior. Details and refinements of the elementary theory are presented and discussed in standard references such as Cook (1), Courant and Friedricks (2), Hirschfelder, Curtiss and Bird (8), and Lewis von Elbe (15), and therefore will not be presented here.

The chemical species which will be present must be specified to formulate the equilibrium conditions. Preliminary calculations indicated that ozone and hydrogen peroxide would be present only in negligible quantities. Therefore, only the six species molecular hydrogen, molecular oxygen, water, atomic hydrogen, atomic oxygen, and hydroxyl were assumed to be present.

When a detonation wave collides with a solid wall, a reflected wave travels back through the burned gases. This reflected wave produces a second pressure rise, and the resulting pressure is often called the impact pressure. The pressure behind the reflected wave was computed from the elementary theory of shock waves with the assumption of no further change in composition across the reflected wave. This assumption is discussed subsequently.

The detailed mathematical representation of the problem is presented in reference 6 .

\section{COMPUTATION PROCEDURE}

In order to minimize interpolation of tabulated thermodynamic data the equations describing the elementary theory of detonation were arranged so that the following procedure could be used to compute the detonation characteristics:
1. Fix initial composition and final pressure.

2. Compute product composition and enthalpy for four assumed final temperatures.

3. Compute the final temperature corresponding to the initial composition, the final pressure, and each of the four product compositions and enthalpies.

4. Compute the error between the four calculated and assumed final temperatures.

5. Employ a Lagrangian four-point interpolation on these errors to determine that temperature for which the error is zero.

6. Employ the same interpolation factors to calculate the final molecular weight and heat-capacity ratio.

7. Calculate the detonation velocity and the initial pressure.

In order to insure that sufficient accuracy was obtained with a four-point interpolation, a single temperature was computed with a five-point interpolation. The difference in final temperature was $2^{\circ} \mathrm{F}$. which is roughly a $0.05 \%$ error. The absence of scatter in the calculated points further verified the accuracy of the procedure.

An IBM-650 computer was used to compute equilibrium compositions for an initial temperature of $298^{\circ} \mathrm{K}$; initial mole fractions of hydrogen of 0.4 , $0.5,0.6,0.6667$, and 0.75 ; final (detonation) pressures of $10,25,50,75$, $100,250,500,750,1,000,1,500$, and $2,000 \mathrm{~atm}$; and product temperatures from $3,000^{\circ}$ to $5,000^{\circ} \mathrm{K}$. The details of the calculation procedure are given in reference 6 . The equilibrium compositions for all of the computed conditions are given in reference 6 and have been placed on file*. Although the equilibrium compositions were obtained as an intermediary in the calculation of detonation velocities, they are of general applicability. The effect of pressure on the equilibrium composition for a stoichiometric mixture is illustrated in Figure 2 for $3,000^{\circ}$ and $5,000^{\circ} \mathrm{K}$.

\section{THE EFFECT OF ERRORS IN PRESSURE SENSITIVE PHYSICAL PROPERTIES ON DETONATION WAVE VELOCITIES}

Specific heats and enthalpies (13) and equilibrium constants $(9,12)$ of the six components as a function temperature at low pressure were utilized in the computations. No data were found for the effect of pressure on these properties or on the compressibility factors and fugacity coefficients

- Tabular material has been deposited as document 6378 with the American Documentation Institute, Photoduplication Service, Library of Congress, Washington 25, D. C., and may be $35-\mathrm{mm}$. microfilm. at the highest pressures for which the computations were made (final pressures of 2,000 atm.). High-pressure data which are available do not extend to the high temperatures which were attained. Even the generalized charts in terms of reduced pressures and temperatures do not extend to these conditions.

In order to determine what errors might be introduced in the computed velocities by the use of idealized properties, the equations describing the wave were reformulated in terms of compressibility factors and fugacity coefficients, and total derivatives of all of the dependent variables were formulated in terms of the partial derivative of the properties. Then the fractional and absolute errors in detonation velocity resulting from fractional and absolute errors in each of the properties were computed for a stoichiometric mixture at a final pressure of 2,000 atm. Estimates of the probable uncertainties in the properties were based primarily on the generalized reduced property charts $(11,19,20)$. The initial compressibility factor was approximated as a mole average of the compressibility factors of molecular hydrogen and molecular oxygen under the initial conditions. The final reduced temperature and pressure of molecular hydrogen were beyond the range of the charts, and the critical properties of atomic hydrogen, atomic oxygen, and hydroxy are not known. Therefore the average compressibility factor of the burned mixture was assumed to be that of molecular oxygen and water, which was roughly 1.1 under the final conditions. Since the deviation of the other four components from ideality is probably less than that of molecular oxygen and water, a maximum error of $-10 \%$ was attributed to the use of a compressibility factor of unity. The other property errors were similarly approximated.

The resulting negative errors in the computed velocity were found to total $9.3 \%$ and the positive errors to total $8.9 \%$, yielding a net error of only $-0.4 \%$. In this case errors in some properties yield an appreciable error in detonation velocity, but the total positive and negative errors very nearly cancel. The detonation velocity is most sensitive to errors in the sensible enthalpies and fugacity coefficients and is quite insensitive to errors in the compressibility-factor and heat-capacity ratios. The estimated net error is very dependent on the arbitrary error attributed to the individual properties. For example the fractional errors in the fugacity coefficients of molecular hydrogen and hydroxyl were assumed to be $-15 \%$. Had they been assumed to be $-10 \%$, the net error in the theoretical 
detonation velocity would have been $-2.3 \%$ instead of $-0.4 \%$. For lower pressures and for product gases containing less water the error introduced by idealized properties would be expected to be less than the above estimate. The details of the error analysis are given in reference 6 and have been placed on file."

\section{RESULTS AND DISCUSSION}

All experimental runs were initiated at room temperatures which varied between $70^{\circ}$ and $80^{\circ} \mathrm{F}$. The experimental and theoretical results of Moyle, Morrison, and Churchill (18) indicate that this small variation has a negligible effect on the detonation characteristics. pressure at discrete mole fractions of hydrogen. With this method, as opposed to cross plotting, the identity of the individual points is preserved. Since the deviations of the actual mole fractions from the nominal values were small and the slopes of the original curves were fairly constant, the uncertainty introduced by translation of the points was very slight. As the mole fraction of hydrogen approaches the limiting rich and lean values for which detonation will occur, the detonation velocity is known to fall off sharply from the almost linear dependence on composition indicated in Figure 3, and these curves should not be extrapolated.

The theoretical velocities correspond to those which would be measured in drogen) these values correspond to less than $1 \%$ and less than $0.013 \%$, respectively, of the measured velocity and hence are negligible for practical purposes. The original and corrected data are on file.

The theoretical and corrected experimental relationships between detonation velocity and initial pressure at various initial mixture compositions are presented in Figures 4 and 5. An inspection of these figures and of the plot of the original experimental data (Figure 3) indicates that the experimental scatter is less than $2 \%$ except for hydrogen-rich mixtures at low pressure. Experimental consistency was not attainable for 0.75 and 0.80 mole fraction hydrogen at 14.5 and $25 \mathrm{lb} . /$

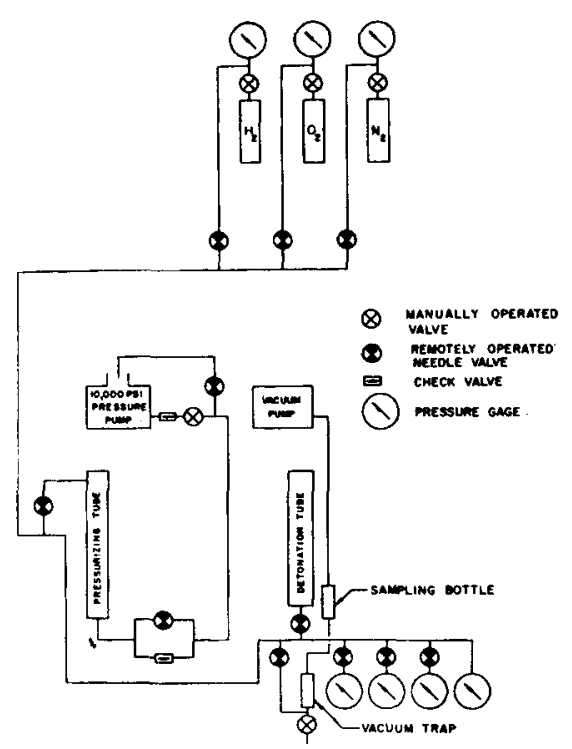

Fig. 1. Experimental apporatus.

Data were taken at initial pressures of $14.4,25,50,100,500$, and 1,000 lb./ sq. in. abs. Velocities at each of these pressures were measured for mixtures having nominal mole fractions of molecular hydrogen of $0.4,0.5,0.6,0.667$, 0.75 , and 0.8 . Because of the method of charging it was difficult to obtain mixtures having the exact compositions mentioned above. Only after the measurements were made and the mixtures analyzed were the initial compositions known.

In order to obtain plots at constant composition the experimental velocities were first plotted vs. mole fraction of hydrogen at the various initial pressures (Figure 3 ), and the experimental points were corrected for their deviation from the nominal hydrogen fraction by translation parallel to curves drawn through the raw data. Then the velocity could be plotted vs. initial

- See footnote on page 501.

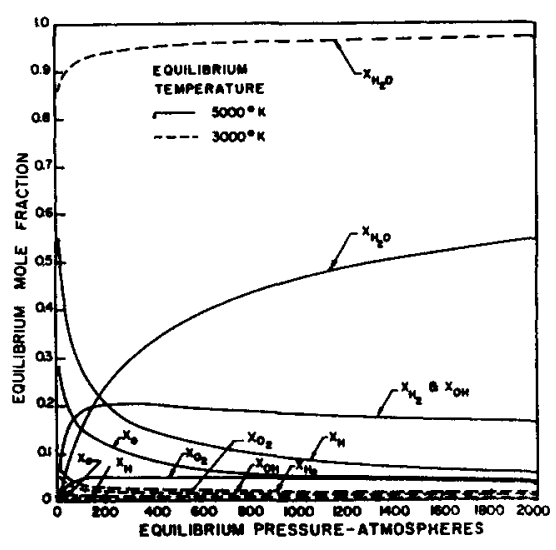

Fig. 2. Equilibrium compositions for a stoichiometric mixture of hydrogen and oxygen.

a tube of infinite diameter. Therefore in order to compare theoretical and experimental velocities it is necessary to correct the experimental measurements for the effect of a finite tube diameter. Fay (5) has recently presented a theoretical explanation for the observation of Kistiakowsky and Zinman (14) and Moyle, Morrison, and Churchill (18) that a plot of detonation velocity vs. the reciprocal of tube diameter results in a straight line. Furthermore Fay indicates that the velocity deficiency due to wall effects should be inversely proportional to the initial pressure.

The measured velocities in feet per second were therefore corrected by adding $30 / D P$, where $D$ is the diameter in inches, $P$ is the pressures in atmospheres, and 30 is an empirical coefficient in ft./ (sec.) (atm.) (in.) taken from the correlation of Moyle, Morrison, and Churchill for the effect of tube diameter in various hydrogenoxygen mixtures at atmospheric pressure. This correction varied from 60 $\mathrm{ft}$./sec. at atmospheric pressure to 0.9 $\mathrm{ft} . / \mathrm{sec}$. at 1,000 lb./sq. in. abs. For the leanest mixture ( $40 \%$ molecular hy-

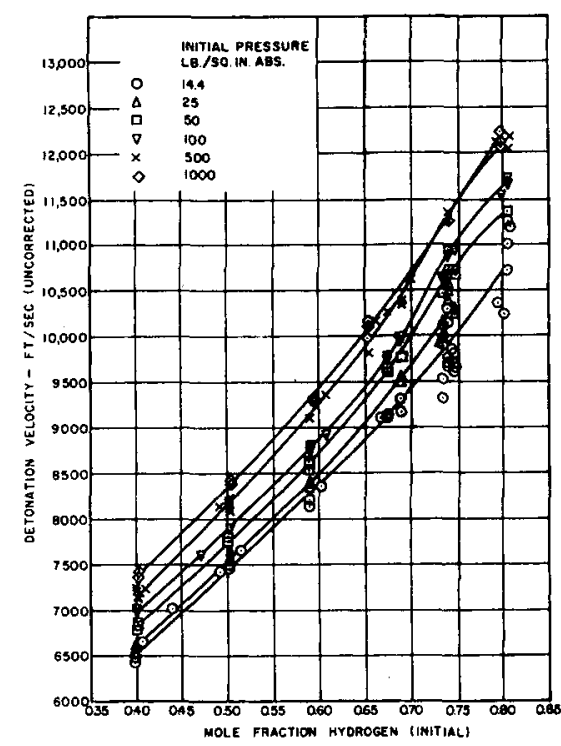

Fig. 3. Experimental detonation velocities.

sq. in. abs. initial pressure. The most probable explanation is that stable detonation did not consistently develop in the alloted distance between the ignitor wire and the first probe (58 in.) for these rich mixtures at low pressure. As the initial pressure was increased to $50 \mathrm{lb} . / \mathrm{sq}$. in. abs. and beyond, reproducible data were obtained even for the richest mixtures.

At low pressure the experimental velocities lie slightly below the theoretical curves. This discrepancy is probably due to oversimplifications in the elementary theoretical model, such as the assumption of a planar discontinuity and chemical equilibrium.

At $1,000 \mathrm{lb} . / \mathrm{sq}$. in. abs. the averages of the various groups of experimental points are about $2 \%$ above the theoretical curves. These deviations are probably due to the use of idealized properties for the computed curves. At high pressure imperfections in the ele-

- See footnote in column 1. 
mentary theory are probably masked by the greater effect of nonideality. Furthermore equilibrium is probably inore closely approached at the high pressure because of the shorter mean free path for chemically productive collisions.

The computed ratios of detonation and impact pressures to the initial pressures are plotted vs. initial pressure in Figure 6. The ratio of pressures across the detonation wave in a stoichiometric mixture increases only from 17.7 to 21.6 as the initial pressure is increased from 8.29 to $1,362 \mathrm{lb} . / \mathrm{sq}$. in. abs. The ratio of pressures across the reflected wave is almost independent of initial composition and initial pressure and averages about 2.5 . The ratio

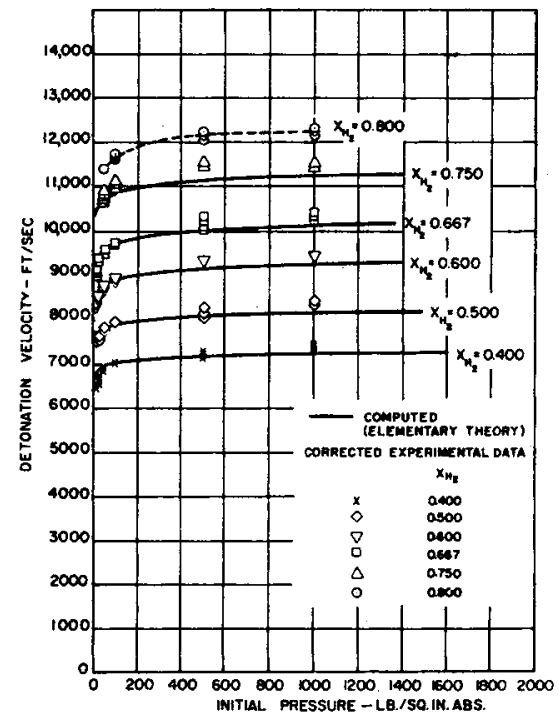

Fig. 4. Comparison of computed and corrected experimental detonation velocities, high-pressure range.

of impact to initial pressure in a stoichiometric mixture increases from 43 to 53 over the above range of initial pressure. The absolute values of the computed detonation and impact pressures for a stoichiometric mixture at an initial pressure of $1,367 \mathrm{lb} . / \mathrm{sq}$. in. abs. are thus 29,390 , and $72,260 \mathrm{lb} . / \mathrm{sq}$. in. abs. Even higher ratios and pressures are obtained if the initial temperature is lowered (18).

Luker, McGill, and Adler (17) computed detonation characteristics for the stoichiometric mixture only, over the same range of pressures, assuming the ideal gas law for the final mixture but correcting the initial density for nonideality. In addition their calculations allowed for a new equilibrium composition behind the reflected wave. Their computed wave velocities agree within $0.5 \%$ with those computed in this investigation. However their computed detonation and reflected pressure ratios are seen in Figure 6 to be $10 \%$ lower than those of this investigation at the nighest initial pressures. The differences in the computed detonation pressures are apparently due to the correction of the initial density for nonideality. The differences in the computed impact pressures are due to the allowance for change in composition across the reflected wave as well as to the correction in the initial density. Taking into account nonideality at both the initial and final conditions may result in a smaller net correction than taking into account nonideality in the initial density only. Hence the relative reliability of the two estimates of developed pressure cannot be resolved until high pressure fugacities and den-

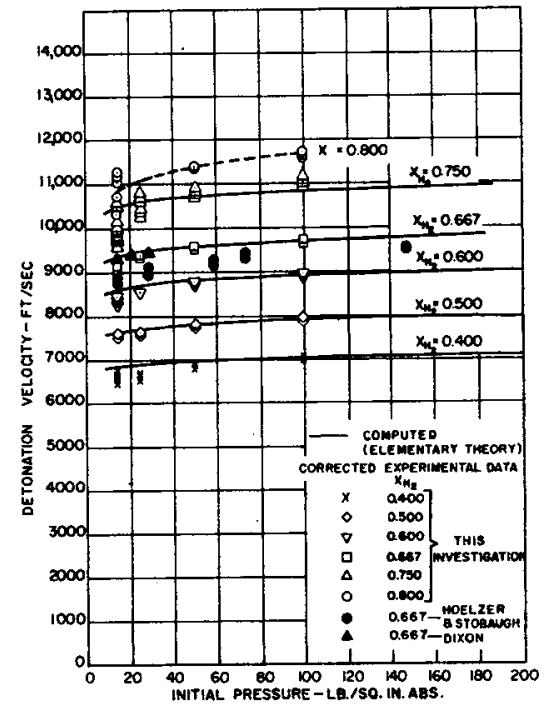

Fig. 5. Comparison of computed and corrected experimental detonation velocities, low-pressure range.

sities are available and are incorporated in the computations. The discrepancies do establish however that the computed pressures are more sensitive than the computed wave velocity to errors in the physical properties and to oversimplifications in the theoretical model.

The excellent agreement between the measured and computed detonation velocities for a wide range of temperatures, pressures, and compositions indicates that the simplified theory of detonation used herein is adequate for the computation of velocity. Experimental data to test the computed detonation and impact pressures are very difficult to obtain, and reliable values are not yet available for even ambient conditions. Transient effects $(1,7)$ and multiple reflections must also be considered if the failure of process equipment due to a detonation is to be predicted. Nevertheless the computed detonation and impact pressures are a first-order guide to the design of equipment in which a detonation could occur. Luker and Leibson (16) have indeed shown that the computed reflected pressure for conditions under which a rupture disk failed under detonation bears a constant ratio slightly greater than unity to the rated static bursting pressure, indicating that use of impact pressures for design is conservative.

\section{DESTRUCTION OF EXPERIMENTAL EQUIPMENT}

It was originally intended to extend the experiments to initial pressures of $10,000 \mathrm{lb} . / \mathrm{sq}$. in. abs. However as a mixture of $80 \%$ molecular hydrogen and $20 \%$ molecular oxygen was being

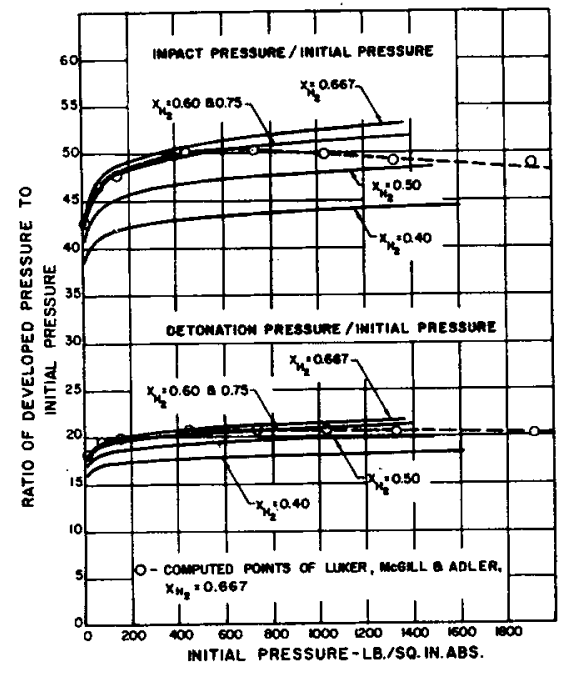

Fig. 6. Computed ratios of detonation and impact pressures to initial pressure.

compressed for a test at 4,000 lb./sq. in. abs., an unintended detonation occurred at $3,500 \mathrm{lb} . / \mathrm{sq}$. in. abs. The auxiliaries, which were all isolated from the detonation tube during controlled detonations and which were not designed for such a pressure, were destroyed. The detonation tube itself, which was designed for such pressures, was undamaged. The cause of this detonation is unknown. This accident suggests caution in future work with hydrogen-oxygen mixtures at high pressures.

\section{CONCLUSIONS}

This investigation indicates that velocities computed from the highly idealized theory of detonation are in reasonable agreement with experimental values throughout an extensive pressure range from 14.4 to $1,000 \mathrm{lb} . / \mathrm{sq}$. in. abs. for hydrogen-oxygen mixtures containing 40 to 80 mole \% molecular hydrogen.

At the lower pressures the slight disagreement between experimental 
and predicted velocities is probably due to oversimplifications in the theoretical model. Inconsistencies in the experimental data for the lowest pressures and richest mixtures are probably due to insufficient distance for wave development.

At higher pressures the slight disagreement can be rationalized in terms of the idealized properties necessarily used in the computations. The effects of errors in the different properties tend to cancel, leaving a small net error in the predicted velocity.

An important consideration for design purposes is the pressure realized when a detonation occurs. The computed ratios of the detonation and impact pressures to the initial pressure are about 20 and 50, respectively, for the conditions investigated.

\section{ACKNOWLEDGMENT}

This work was sponsored in part by Project SQUID which is supported by the Office of Naval Research, Department of the Navy, under contract Nonr 1858 (25) NR-098-038. The experimental work was carried out at the Aircraft Propulsion Laboratory of the University of Michigan. The support and advice of R. B. Morrison,
T. C. Adamson, D. L. Katz, D. V. Ragone, and Alexander Weir, Jr., are appreciated.

\section{LITERATURE CITED}

1. Cook, M. A., "The Science of High Explosives," Reinhold, New York (1958).

2. Courant, R., and K. O. Friedrichs, "Supersonic Flow and Shock Waves," Interscience, New York (1948).

3. Dixon, H. B,, Phil. Trans. Roy. Soc., A184, 97 (1893); A200, 315 (1903).

4. Dumanois, P., and P. Laffitte, Compt. rend, 183, 284 (1926).

5. Fay, J. A., Physics of Fluids, 2, 283 (1959).

6. Gealer, Roy L., Ph.D. thesis, Univ. Mich., Ann Arbor (1958).

7. Gordon, W. E., A. J. Mooradian, and S. A. Harper, "Seventh Symposium (International) on Combustion," $p$. 752, Butterworth, London, England (1959).

8. Hirschfelder, J. F., C. F. Curtiss, and R. B. Bird, "Molecular Theory of Gases and Liquids," Wiley, New York (1954).

9. Hirschfelder, J. O., F. T. McClure, C. F. Curtiss, and D. W. Osborne, Abs. Bib. Sci. and Ind. Reps., 2, 743 (1946).

10. Hoelzer, C. A., and W. K. Stobaugh, Thesis, Air University Command,
USAF Inst. Tech., Wright-Patterson Air Force Base, Ohio (1954).

11. Hougen, O. A., and K. M. Watson, "Chemical Process Principles," Part II, Wiley, New York (1949).

12. Huff, V. N., and C. S. Calvert, Nat. Advisory Comm. Aeronaut. Tech. Note 1653 (1948).

13. Huff, V. N., S. Gordon, and V. E. Morrell, ibid., Rept. 1037 (1951).

14. Kistiakowsky, G. B., and W. G. Zinman, "Second ONR Symposium on Detonation," p. 80, Washington, D.C., (Feb. 9-11, 1955).

15. Lewis, Bernard, and Guenther von Elbe, "Combustion, Flames, and Explosions of Gases," Academic, New York (1951).

16. Luker, J. A., and M. J. Liebson, J. Chem. Eng. Data, 4, 133 (1959).

17. Luker, J. A., P. L. McGill, and L. B. Adler, ibid., 136 (1959).

18. Moyle, M. P., R. B. Morrison, and S. W. Churchill, A.I.Ch.E. Journal, 6, 92 (1960).

19. Nelson, L. C., and E. A. Obert, Trans. Am. Soc. Mech. Engrs., 76, 1057 (1954).

20. Perry, J. H., "Chemical Engineers' Handbook," McGraw-Hill, New York (1950).

Manuscript received August 3, 1959; revision received December 28, 1959; paper accepted Jantury 4, 1960. Paper presented at A.I.Ch.E. San Francisco meeting.

\title{
A Scintillation Method for Determining
}

\section{Liquid - Liquid Interfacial Areas}

\author{
GEORGE J. MITSIS, RICHARD R. PLEBUCH, and KENNETH F. GORDON
}

The University of Michigan, Ann Arbor, Michigan

\begin{abstract}
of the interfacial area for ten configurations.
Data for mass transfer between dispersed phases in equipment such as extractors, distillation columns, and sprayers can be correlated on the basis of equations of the form
\end{abstract}

In stirred beakers the interfacial areo between xylene containing a scintillator and tritioted water was measured as a function of stirrer speed by a scintillation counting technique. Because of the $6 \mu$ average range of the tritium betas the rate at which flashes are detected by a phototube is a function of the interfacial area. A calibration curve of count rate against known interfacial area for unstirred phases in containers of various diameters gave a numerical estimate

$$
W=k^{\prime} a\left(f_{s}-f_{w}\right)=k a\left(c_{s}-m c_{w}\right)
$$

Because of the difficulty of obtaining interfacial areas it is usual not to attempt to separate $k$ and $a$, obtaining values for each, but rather to correlate $k a$. Measurements of the interfacial area should contribute to a further understanding of mass transfer.

Interfacial areas have been measured 